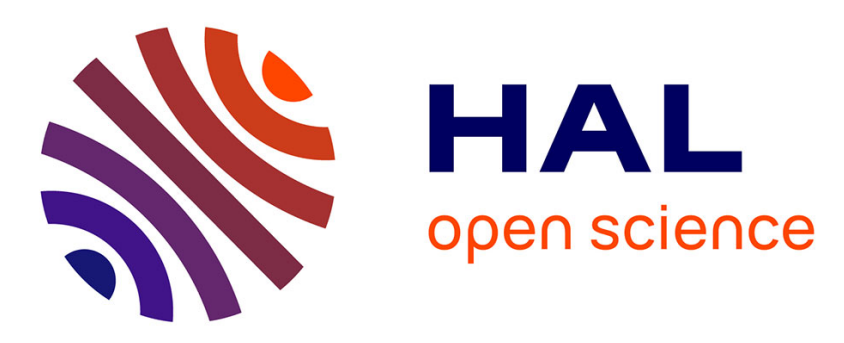

\title{
Finite Blocklength Information Theory: What is the Practical Impact on Wireless Communications?
}

\author{
Philippe Mary, Jean-Marie Gorce, Ayşe Ünsal, H. Vincent Poor
}

\section{To cite this version:}

Philippe Mary, Jean-Marie Gorce, Ayşe Ünsal, H. Vincent Poor. Finite Blocklength Information Theory: What is the Practical Impact on Wireless Communications?. 2016 IEEE Global Communications Conference: Workshops: First IEEE International Workshop on Low-Layer Implementation and Protocol Design for IoT Applications, Dec 2016, Washington, DC, United States. hal-01390904

\section{HAL Id: hal-01390904 https://hal.science/hal-01390904}

Submitted on 8 Nov 2016

HAL is a multi-disciplinary open access archive for the deposit and dissemination of scientific research documents, whether they are published or not. The documents may come from teaching and research institutions in France or abroad, or from public or private research centers.
L'archive ouverte pluridisciplinaire HAL, est destinée au dépôt et à la diffusion de documents scientifiques de niveau recherche, publiés ou non, émanant des établissements d'enseignement et de recherche français ou étrangers, des laboratoires publics ou privés. 


\title{
Finite Blocklength Information Theory: What is the Practical Impact on Wireless Communications?
}

\author{
Philippe Mary*, Jean-Marie Gorce ${ }^{\dagger}$, Ayse Unsal ${ }^{\dagger}$, H. Vincent Poor $^{\ddagger}$ \\ *INSA de Rennes, IETR UMR CNRS 6164, \\ Email: philippe.mary@insa-rennes.fr \\ †Université de Lyon, INSA, INRIA \\ $\ddagger$ Electrical Engineering Dept, Princeton University
}

\begin{abstract}
Recent advances in information theory have provided achievability bounds and converses for the coding rate for the finite blocklength regime. In this paper, recent results on the non-asymptotic coding rate for fading channels with no channel state information at the transmitter are exploited to analyze the goodput in additive white Gaussian noise (AWGN) and the energy-efficiency spectral-efficiency (EE-SE) tradeoff where the fundamental relationship between the codeword length and the EE is given. Finally, the true outage probability in Ricean and Nakagami- $m$ block fading channels is investigated and it is proved that the asymptotic outage capacity is the Laplace approximation of the average error probability in finite blocklength regime.
\end{abstract}

\section{INTRODUCTION}

The channel capacity is the maximal achievable rate for a given average/maximum error probability in the asymptote of infinite codeword length. This fundamental communication limit was derived by Shannon for various channels such as the discrete memoryless channel (DMC) and the additive white Gaussian noise (AWGN) channel [1]. The fundamental performance evaluation in block fading channels encounters a definition problem, at least in the Shannon sense. Indeed, the channel capacity of block fading channels is strictly zero in the Shannon sense since no codeword can be designed to ensure a vanishing error probability for all the channel realizations, when channel state information (CSI) is not available at the transmitter. The outage capacity is defined as the maximal spectral efficiency achievable under a given error probability; see [2] and references therein. However, the term 'capacity' is not really well chosen since it is neither an upper nor a lower bound but simply an asymptotic approximation of the successful packet transmission rate.

The behavior of the second-order rate, i.e. achievable rate for finite blocklength, with respect to (w.r.t.) the asymptotic channel capacity is an old fundamental problem dating back to Feinstein and Gallager in the 1960's [3], [4]. Recently, Polyanskiy et al. reformulated the problem and rigorously derived some non-asymptotic achievability and converse results for the finite blocklength regime [5]. This work motivated considerable interest and several authors have continued the characterization of the non-asymptotic achievable rate regions for more complicated channels such as the multiple access channel (MAC) and broadcast channel (BC), e.g. [6], [7], [8], [9].

The finite blocklength theory is particularly relevant when addressing the block fading channel since a finite codeword length spans one realization of the channel. Several works have dealt with non-asymptotic achievability and converse bounds in block fading environments [10], [11], [12], [13]. In terms of context, the non-asymptotic information theory is particularly relevant to address the upcoming challenges of internet of things (IoT) and machine-to-machine (M2M) communications which will be part of the $5 \mathrm{G}$ networks. Indeed, in these networks, the flow is expected to be sporadic and with a relatively small quantity of information to transmit.

In this paper, we exploit recent results on the nonasymptotic rate for fading channel with no channel state information at transmitters (CSIT) to investigate the impact of the finite blocklength on physical parameters of practical systems, such as the energy-efficiency (EE) or packet error rate (PER). Some studies have dealt with the finite blocklength framework to analyze some practical communication schemes, e.g. [14], in which authors have used the recent finite blocklength information theory results to derive closed-form expression for the outage probability and feedback delay of incremental redundancy (IR) hybrid automatic repeat request (HARQ).

The rest of this paper is organized as follows. Section II provides the model and definitions used throughout the paper. Section III deals with the fundamental behavior of the goodput and EE of a finite blocklength communication system. The average error probability in block fading channels is addressed in Section IV. Simulation results are given in Section V and conclusions are drawn in Section VI.

\section{SYSTEM MODEL AND ASSUMPTIONS}

We consider a wireless network in which the nodes exchange small packets, typically $k \leq 100$ where $k$ is the number of bits in each packet. From an information theoretic perspective, these packets are considered as messages. Let $M=2^{k}$ denote the cardinality of the message space. An encoder is a mapping function such as $f:\{1, \cdots, M\} \rightarrow \mathcal{A}^{n}$, where $\mathcal{A}^{n}$ is the set of codewords of length $n$, and $n$ is also known as the number of channel uses (c.u.) in transmitting a packet. The blockcode is transmitted over a noisy, possibly block fading, channel which corrupts the bits. At the receiver end, a decoder produces an estimate of the original message by observing the channel output, according to a certain function given as $g: \mathcal{B}^{n} \rightarrow\{1, \cdots, M\}$, where $\mathcal{B}^{n}$ is the set of received codewords of length $n$. The average error probability is defined as $\epsilon=\operatorname{Pr}(W \neq \hat{W})$, where $W \in\{1, \cdots, M\}$ is the message to be sent. According to [5], a tuple $(n, M, \varepsilon)$ is feasible, if 
a source of cardinality $M$ can transmit with a probability of success $1-\varepsilon$ over $n$ channel uses.

Definition 1. An AWGN channel is defined as a triple of two alphabets, i.e. $\mathcal{A}=\mathbb{C}^{n}, \mathcal{B}=\mathbb{C}^{n}$, and a collection of conditional probability functions, i.e. $P_{Y^{n} \mid X^{n}}$ representing the channel such as

$$
P_{Y^{n} \mid X^{n}=x^{n}}=\mathcal{N}\left(x^{n}, I_{n}\right)
$$

where $I_{n}$ is the $n \times n$ identity matrix and $X^{n}, Y^{n}$ represent the channel inputs and outputs, respectively, over $n$ channel uses.

The codewords are subject to an equal-power constraint, i.e. for each codeword $c_{i} \in X^{n}, i \in\{1, \cdots, M\}$, we have $\left\|c_{i}\right\|^{2}=n P=E$, where $P$ and $E$ are the power per channel use and the energy of the code, respectively. In the nonasymptotic regime, the error probability cannot be as small as desired for a given rate $k / n$, as stated in [5]. The authors of [5] have shown that for an error probability (maximal or average) $\epsilon$ and a power constraint $P$, the following approximation on the achievable rate holds:

$$
\frac{1}{n} \log _{2} M(n, \epsilon, P) \approx C(P)-\sqrt{\frac{V(P)}{n}} Q^{-1}(\epsilon)
$$

considering that the noise variance $\sigma^{2}=1$, we have $C(P)=\log _{2}(1+P)$ the Shannon channel capacity, $V(P)=$ $\frac{P}{2} \frac{P+2}{(P+1)^{2}} \log _{2}^{2} e$ is the AWGN channel dispersion and $Q(x)=$ $1 / \sqrt{2 \pi} \int_{x}^{\infty} \exp \left(-u^{2} / 2\right) d u$. The non asymptotic achievable rate in (2) reveals to be tight even for relatively small $n$, e.g. $n=200$ [5].

\section{GOODPUT AND ENERGY EFFICIENCY IN AWGN CHANNEL}

\section{A. Goodput}

As already mentioned, for non-asymptotic values of $n$, the achievable data rate for a given error probability, denoted by $R(n, \epsilon)$, can be very far from the asymptotic Shannon limit. In a practical system, four parameters $k, n, \varepsilon, P$ can be tuned to optimize the performance. Let us consider a system where the packet size is fixed, which means that $n$ channel uses are needed to transmit an amount of information. Under this assumption, what is the best choice to maximize the global capacity and how far is the result from the Shannon capacity? The goodput, $\mathcal{G}(k \mid n, P)$, for a given $k$ knowing the number of c.u. and power per c.u. is defined as

$$
\mathcal{G}(k \mid n, P)=(1-\varepsilon(k \mid n, P)) \cdot \frac{k}{n}
$$

where $\epsilon(k \mid n, P)$ is the average error probability for $k$ information bits sent in $n$ c.u. with a power $P$ per c.u. Figure 1 shows the goodput defined as in (3) versus the rate, i.e. the number of information bits $k$ when $n$ is fixed. It can be observed that for low rates, the goodput matches with the rate (according to Shannon) since very low error probability can be achieved. However, for rates near capacity, i.e. $1 \mathrm{bit} / \mathrm{s} / \mathrm{Hz}$ at $P=0 \mathrm{~dB}$ (for unitary noise variance), the goodput suddenly decreases as a consequence of the Gaussian $\mathrm{Q}$ function involved in the error probability, i.e. $Q(\sqrt{n / V(P)}(C(P)-k / n))$. In particular, we note that for

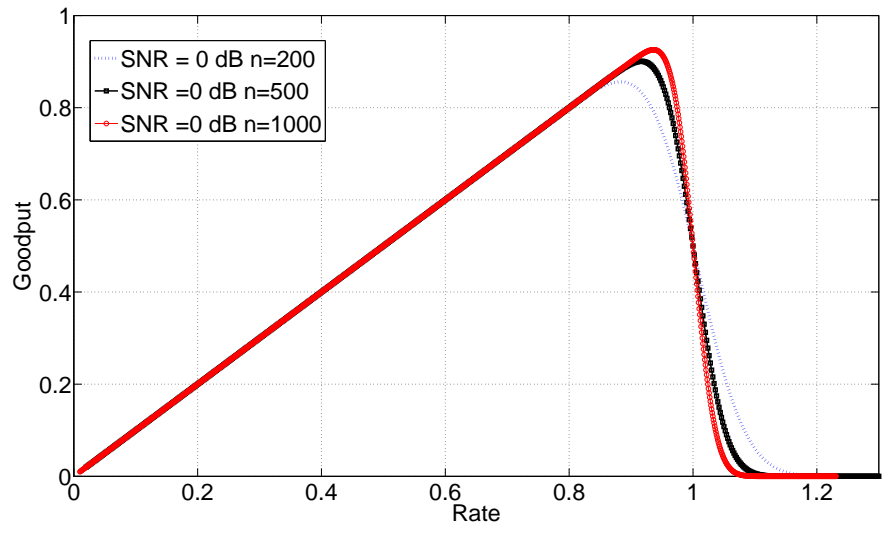

Fig. 1. The numerical evaluation of the goodput given by (3) for three different values of the blocklength $n$

short packets, the goodput is only $50 \%$ of the achievable channel capacity, which means that working with the Shannon limit is clearly far from the reality for small packet communications. The optimal number of information bits leading to the maximum achievable goodput can be easily obtained by solving $\frac{\partial \mathcal{G}}{\partial k}=0$.

\section{B. Energy efficiency versus spectral efficiency}

The energy efficiency - spectral efficiency (EE-SE) tradeoff is a Pareto front drawing the achievable frontier of a wireless communication system on these two quantities. It is an important information especially when the energy efficiency comes into play in the design of a wireless communication system as it is the case for IoT for instance. In order to evaluate different EE-SE tradeoffs, for a given quantity of information $k$ to be sent with a given reliability $\varepsilon$, we are interested in trading between $n$ and $E=n \cdot P$. In the asymptotic case, the relation between capacity and energy is simply given by $k / n=\log _{2}(1+E / n)$ which leads to the classical Pareto front: $E=\left(2^{\frac{k}{n}}-1\right) \cdot n$, with $k=\log _{2}(M)$. In this approach, there is no error probability since the asymptotic Shannon capacity is assumed to be achievable and the idea is to say that if the rate exceeds the capacity all packets are dropped. However, especially with small packets and poor coding, the Shannon capacity is not achieved. At any signal level, the system will suffer from a certain error probability and hence the EE-SE tradeoff will be different from that in the asymptotic case. From (2) we have:

$$
k=n \cdot C(E / n)-\sqrt{n \cdot V(E / n)} Q^{-1}(\epsilon)
$$

We can now state the first main result:

Theorem 1. The energy efficiency - spectral efficiency tradeoff subject to the packet size and error probability constraints is given by:

$$
\begin{aligned}
E(\lambda) & =f^{2}(\lambda) \cdot V^{-1}(\lambda) \\
n(\lambda) & =f^{2}(\lambda)
\end{aligned}
$$




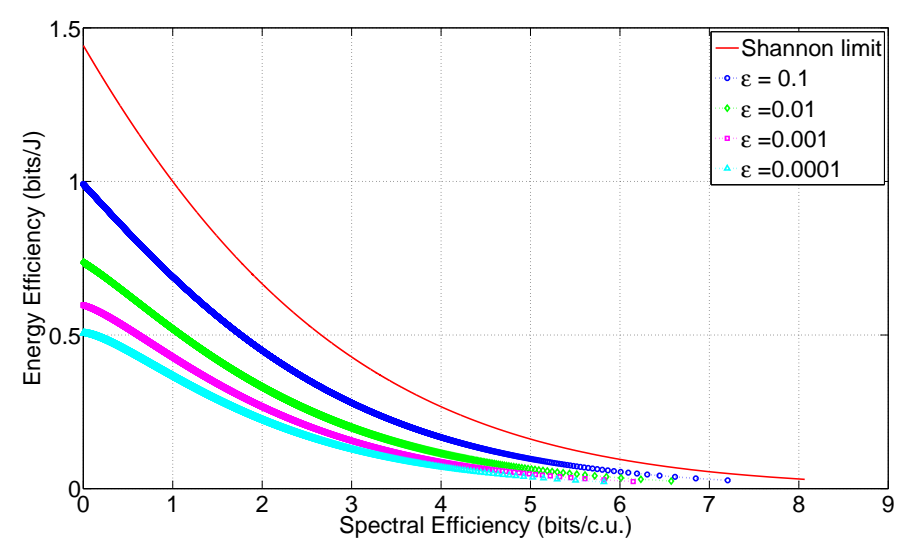

Fig. 2. EE-SE tradeoff for $k=16$ bits and error probability ranging from $10^{-1}$ to $10^{-4}$.

where $\lambda \in\left[0 ; \log _{2}^{2}(e) / 2[\right.$ and

$$
\begin{aligned}
V^{-1}(\lambda) & =\frac{1}{\sqrt{1-\frac{2 \lambda}{\log _{2}^{2}(e)}}}-1 \\
f(\lambda) & =\frac{b(\lambda)+\sqrt{b^{2}(\lambda)+4 k C\left(V^{-1}(\lambda)\right)}}{2 C\left(V^{-1}(\lambda)\right)} \\
b(\lambda) & =\sqrt{\lambda} \cdot Q^{-1}(\varepsilon)
\end{aligned}
$$

Proof: The proof relies first on the inversion of the divergence function $\lambda=V(E / n)$ which is a second order polynomial with a unique positive solution : $E / n=V^{-1}(\lambda)=$ $1 / \sqrt{1-2 \lambda / \log _{2}^{2}(e)}-1$. Since $\mathrm{V}$ is a monotonic function in $\left[0, \log _{2}^{2}(e) / 2[, \lambda\right.$ is bounded. Replacing $E / n$ in (4) leads to a new polynomial $n C\left(V^{-1}(\lambda)\right)-\sqrt{n} \cdot b(\lambda)-k=0$ which has a unique positive solution in $\sqrt{n}$. This completes the proof of Theorem 1 .

Let us fix the number of information bits, $k$, to be transmitted. The spectral and energy efficiencies are simply $k / n$ and $k / E$ where $n$ and $E$ are given by (5) and (6) respectively. Figure 2 presents the EE-SE tradeoff for $k=16$ bits and various values of codeword error probability, i.e. from $\epsilon=10^{-1}$ to $\epsilon=10^{-4}$. It can be noticed that the real frontier can be relatively far from the Shannon bound when dealing with small packets as is the case in IoT networks. Moreover, the more the targeted error rate decreases the more the SE-EE tradeoff in finite length theory mismatches with the Shannon bound.

\section{OUTAGE PROBABILITY IN BLOCK FADING}

\section{A. Model}

We consider block fading channels, where the state is not known at the transmitter [15]. The signal model is given as

$$
y[l]=h[l] x[l]+w[l]
$$

where the index $l$ denotes the $l$-th coherence interval, $w \sim$ $\mathcal{C N}(0,1)$ is centered complex AWGN with unit variance, $x$ is the transmitted codeword and $h$ is the complex channel coefficient, being Rice or Nakagami- $m$ distributed with specular component $K$, or parameter $m$ respectively ${ }^{1}$. The channel remains constant over $T$ consecutive symbols and a codeword of length $n=T$ spans over one channel realization.

\section{B. Message error rate in fading channels}

The block-fading channel is conditionally ergodic w.r.t. the channel realization $h$. In that case, (2) holds in non-asymptotic regime by setting

$$
\begin{aligned}
C(\gamma) & =\log _{2}(1+\gamma) \\
V(\gamma) & =\frac{\gamma(\gamma+2)}{2(\gamma+1)^{2}} \log _{2}^{2} e
\end{aligned}
$$

where $\gamma=P|h|^{2}$ and $P$ is the average power per channel use. Since, the error probability extracted from (2) is conditioned on the channel realization, one can define an average error probability in block fading channel as

$$
\bar{\epsilon}=\int_{\mathbb{R}^{+}} Q\left(\sqrt{\frac{n}{V(\gamma)}}(C(\gamma)-R)\right) p_{\gamma}(\gamma) d \gamma
$$

where $p_{\gamma}$ is the probability density function (pdf) of the instantaneous SNR $\gamma$. In some cases, the non-asymptotic average error probability can be different from the outage probability defined with the Shannon formula as

$$
\begin{aligned}
\mathrm{P}(O) & =\mathrm{P}\left(\log _{2}(1+\gamma)<R\right) \\
& =\mathrm{P}\left(\gamma<2^{R}-1\right) \\
& =F_{\gamma}\left(2^{R}-1\right)
\end{aligned}
$$

where $F_{\gamma}$ is the cumulative distribution function (cdf) of the instantaneous SNR. The difference between (14) and (13) is that the former is the integration of the channel probability density function (pdf) weighted by an indicator function (hence null after a threshold, i.e. $2^{R}-1$ ) while the latter is the integration of the pdf weighted by a Gaussian $\mathrm{Q}$ function which is never zero. Hence, according to the nature of the pdf and the rate threshold considered, the average non-asymptotic error probability can be quite different from the outage probability.

There is not an exact closed-form expression for (13), but an interesting question would be "can we approximate this integral?". The average symbol error probability expression combines the Gaussian Q function and the SNR pdf and the Laplace approximation has been shown to be a successful method to approach the exact error rate in fading channels [16], [17], [18]. One can attempt to follow the same kind of steps here, but due to the form of the average error probability one can prove that the Laplace approximation of (13) results in the outage probability obtained with Shannon formula as stated in the following result.

Theorem 2 (Average Error Probability). In block fading channels, the asymptotic outage probability constitutes the Laplace approximation, a.k.a. the saddle point approximation, of the non-asymptotic average error probability, i.e.

$$
\bar{\epsilon} \approx F_{\gamma}\left(\gamma_{0}\right)
$$

\footnotetext{
${ }^{1}$ The Rayleigh distribution is encompassed in the Rice distribution by setting $K=0$ and in the Nakagami distribution with $m=1$.
} 
where $\gamma_{0}=2^{R}-1$ and $F_{\gamma}$ is the cdf of the SNR. For Rice and Nakagami-m channels, $F_{\gamma}\left(\gamma_{0}\right)=1-Q_{1}\left(\sqrt{2 K}, \sqrt{2 \frac{1+K}{\bar{\gamma}} \gamma_{0}}\right)$ and $F_{\gamma}\left(\gamma_{0}\right)=\frac{1}{\Gamma(m)} \gamma_{i n c}\left(m, m \gamma_{0} / \bar{\gamma}\right)$ respectively, where $Q_{1}(\cdot, \cdot)$ is the generalized Marcum $Q$ function of order 1 , $\gamma_{\text {inc }}(a, b)$ is the incomplete gamma function and $\bar{\gamma}$ is the average SNR per c.u.

Proof: Using integration by parts, the expression in (13) can be rewritten as

$$
\bar{\epsilon}=-\int_{\mathbb{R}^{+}} \frac{\partial}{\partial \gamma} Q\left(\sqrt{\frac{n}{V(\gamma)}}(C(\gamma)-R)\right) F_{\gamma}(\gamma) d \gamma
$$

The derivative of the Gaussian $\mathrm{Q}$ function results in

$$
\frac{\partial Q}{\partial \gamma}=-\sqrt{\frac{n}{\pi}} h(\gamma) e^{-T g(\gamma)}
$$

where $h(\gamma)=\sqrt{\frac{1}{\gamma(\gamma+2)}}\left(1-\frac{C(\gamma)-R}{\gamma(\gamma+2) \log _{2} e}\right)$ and $T=\sqrt{\frac{n}{2}}$, and hence the average error probability can be expressed as

$$
\bar{\epsilon} \approx \sqrt{\frac{n}{\pi}} \int_{\mathbb{R}^{+}} h(\gamma) F_{\gamma}(\gamma) e^{-T g(\gamma)}
$$

by letting $\tilde{h}(\gamma)=h(\gamma) F_{\gamma}(\gamma)$ and $g(\gamma)=\frac{(C(\gamma)-R)^{2}}{V(\gamma)}$. The function $g$ is twice differentiable, smooth and has a unique minimum over $\mathbb{R}^{+}$at $\gamma_{0}$. Moreover, $\tilde{h}$ is continuous with a constant sign over $\mathbb{R}^{+}$. Hence, (17) can be approached via the Laplace method with

$$
\bar{\epsilon} \approx \sqrt{\frac{n}{\pi}} \sqrt{\frac{2 \pi}{T g^{\prime \prime}\left(\gamma_{0}\right)}} \tilde{h}\left(\gamma_{0}\right) e^{-T g\left(\gamma_{0}\right)}
$$

Here we obviously have $\gamma_{0}=2^{R}-1$ and using straightforward calculus we obtain $g^{\prime \prime}\left(\gamma_{0}\right)=4 /\left(\gamma_{0}\left(\gamma_{0}+2\right)\right)$ and $\tilde{h}\left(\gamma_{0}\right)=$ $\sqrt{1 /\left(\gamma_{0}\left(\gamma_{0}+2\right)\right)} F_{\gamma}\left(\gamma_{0}\right)$. Substituting these expressions into (18) completes the proof.

The speed of convergence of the second-order average error probability to the actual outage probability in Rayleigh block fading channels is quite fast [10] but the difference is not negligible in Ricean fading channels as we will see in the next section.

\section{NUMERICAL RESULTS}

In this section, the average error probability in the finite blocklength regime is compared to the outage probability in Ricean and Nakagami- $m$ block fading channels. The parameter settings are $K=15 \mathrm{~dB}, m=6$ and $n=200$ throughout this section, unless otherwise mentioned. Recall that the larger $K$ respectively $m$, the more the channel acts as an AWGN channel. Figures 3(a) and 3(b) show the average error and outage probabilities for low and high rates in a Ricean fading channel respectively. A substantial difference can be observed for very low rate, i.e. $R=2 \cdot 10^{-2}$ between the outage probability defined using the Shannon formula and the nonasymptotic average error probability. The practical scenario corresponding to this regime could be wireless communication with a line of sight situation but with a low SNR. In that case, high redundancy in the channel coding is needed to ensure reliable communication. Even for moderate rate, i.e. $R=0.2$ the mismatch between the asymptotic outage probability and

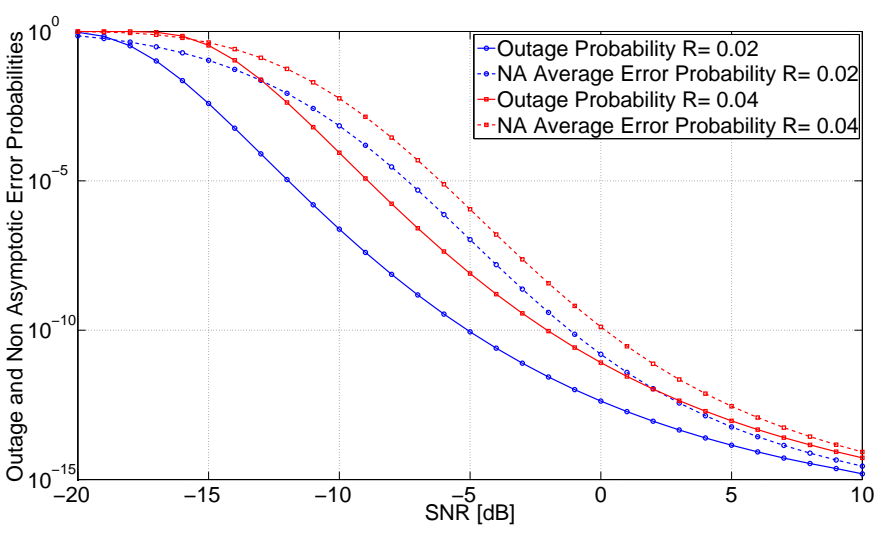

(a) $n=200, K=15 \mathrm{~dB}$ and $R=2 \cdot 10^{-2}$ or $R=4 \cdot 10^{-2}$

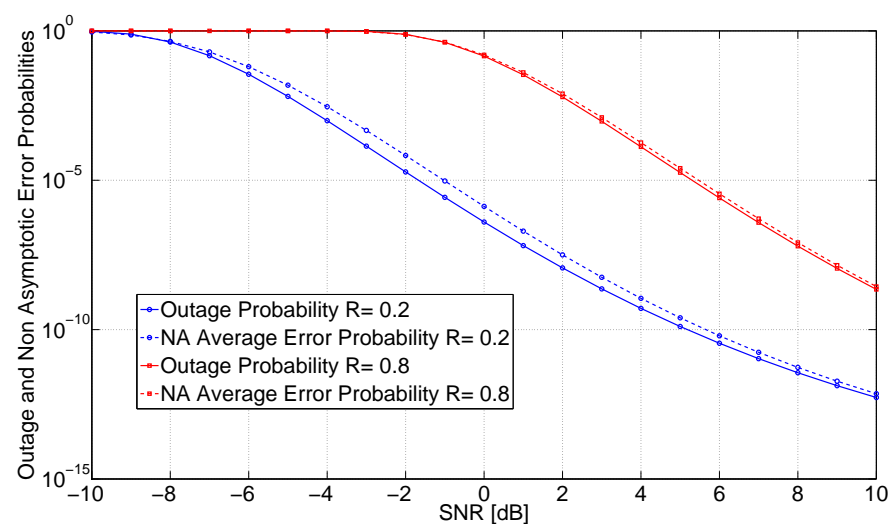

(b) $n=200, K=15 \mathrm{~dB}$ and $R=0.2$ or $R=0.8$

Fig. 3. Average error probability in Ricean fading from non-asymptotic and asymptotic formula.

the average probability could be non-negligible in particular for $\bar{\gamma}=0 \mathrm{~dB}$. For higher rates, e.g. $R=0.8$, the average error and the asymptotic outage probabilities converge as can be seen in Figure 3(b). Moreover for high average SNR, the outage and the average error probabilities converge.

Figures 4(a) and 4(b) show the non-asymptotic average error probability and the outage probability in Nakagami- $m$ fading for low and high coding rates respectively. As in the former case, the greatest difference between the outage probability defined with the Shannon formula and the nonasymptotic average error probability occurs for small rates, i.e. $R=2 \cdot 10^{-2}-4 \cdot 10^{-2}$. Unlike the Ricean fading channel, the gap between the non-asymptotic average error probability and outage probability is asymptotically constant, i.e. for large SNR, due to the linear behavior of error probability in Nakagami $-m$ channels when SNR goes to infinity. In Figure 4(b), the gap between the non-asymptotic average error probability and the outage probability decreases as the coding rate increases due to the weighting of the tail of channel pdf by the Gaussian Q-function. Indeed, the larger $R$, the farther, in SNR, the inflection point of $Q$ function. Hence, the contribution of the integral in the range $\left[\gamma_{0}, \infty\right.$ [ to the non asymptotic error probability is negligible compared to the part in $\left[0, \gamma_{0}\right]$.

In order to analyze how the outage and the finite blocklength average error probabilities behave relative to each other, 


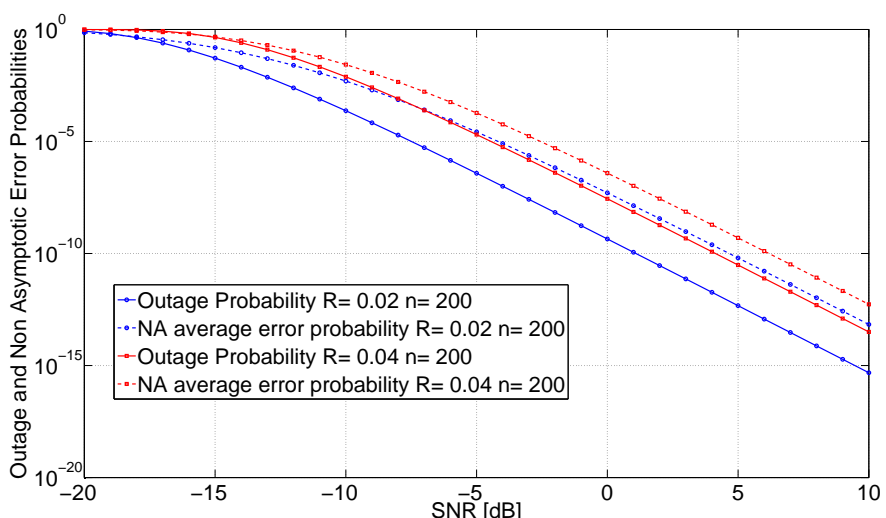

(a) $n=200, m=6$ and $R=2 \cdot 10^{-2}$ or $R=4 \cdot 10^{-2}$

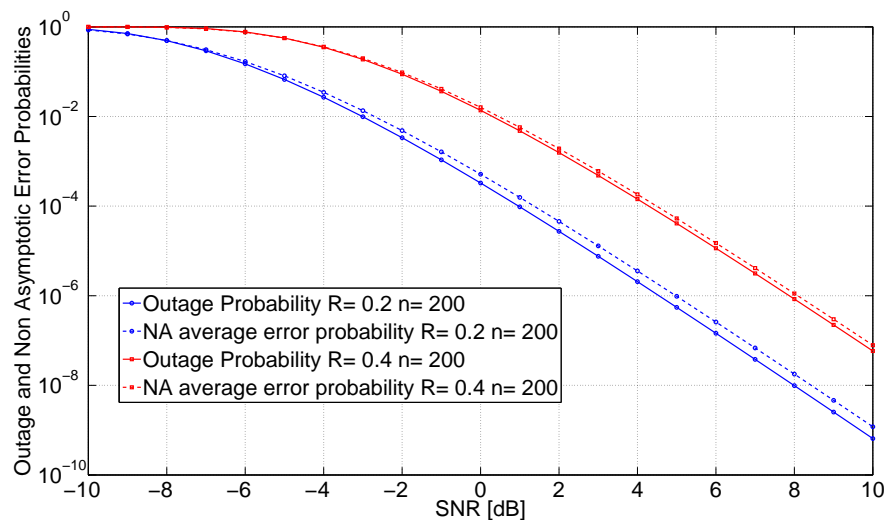

(b) $n=200, m=6$ and $R=0.2$ or $R=0.4$

Fig. 4. Average error probability in Nakagami $-m$ fading from nonasymptotic and asymptotic formula.

we define a mismatch coefficient

$$
Q_{f}=\left|\frac{\bar{\epsilon}-P(O)}{P(O)}\right|
$$

where $P(O)$ is the asymptotic outage probability defined as $P(C(\gamma)<R)$. Figures 5(a) and 5(b) illustrate the behavior of the quality factor defined above according to the average received SNR $\bar{\gamma}$ and the communication rate $R$ for a codeword length $n=200$ in Ricean fading. The colored curves represent the set of couples $(\bar{\gamma}, R)$ for which there is the same amount of relative error between the asymptotic outage probability and the average error probability in block fading channels. Figure 5 (a) in particular illustrates a significant degree of mismatch for small average SNR, i.e. from -12 to $-4 \mathrm{~dB}$, and small rates. In that case, the performance of small packet-based communications has to be assessed by the finite blocklength theory due to the important difference between the asymptotic description and the finite length predictions. In Figure 5(b) a relative error of at least $50 \%$ can be observed for a wide range of rates and SNR values, i.e. from $R=0.2$ to $R=0.7$ and $\bar{\gamma}=-7$ to $\bar{\gamma}=9 \mathrm{~dB}$ respectively.

Figures 6(a) and 6(b) investigate the quality factor defined above in Nakagami- $m$ fading. We can observe the constant behavior of each curve above a certain SNR level, in particular on Figure 6(a). This is due to the constant gap between both metrics in Nakagami fading. Even for high rates, ie. Figure 6(b), the relative difference can be large, at least $10 \%$, for a wide range of combinations of SNRs and rates.

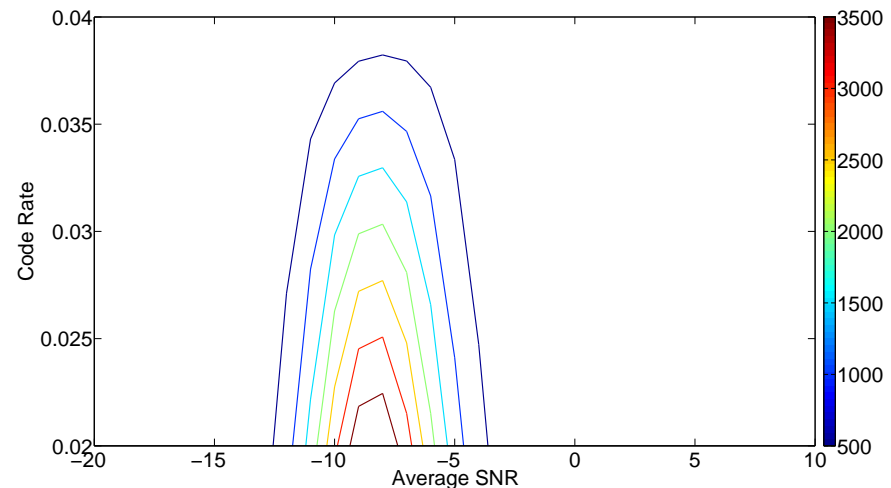

(a) $n=200, K=15 \mathrm{~dB}$, low rates

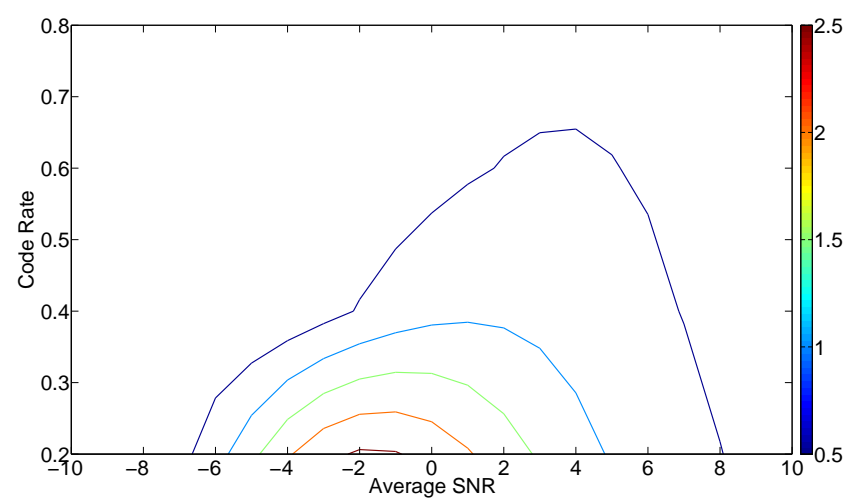

(b) $n=200, K=15 \mathrm{~dB}$, moderate to high rates

Fig. 5. Relative difference between the outage and the average error probabilities in Ricean block fading vs the SNR and the rate.

In these figures, an important change of scale can be observed between low rate and high rate figures. This can be explained by the outage and non asymptotic error probabilities expressions. The outage probability is obtained by integrating the channel pdf up to $\gamma_{0}=2^{R}-1$, which is close to zero for low rates and which increases when rate increases. On the other hand, an integral on $\mathbb{R}^{+}$is involved in the computation of the non asymptotic error probability in which the channel pdf is weighted by the $Q$ function. For low rates, a little part of the channel pdf comes into play in the outage computation contrarily to the non asymptotic error probability formula which is done over $\mathbb{R}^{+}$. Even if the channel pdf is weighted by the $Q$ function, the integration part over $\left[\gamma_{0}, \infty\right.$ [ counts for an important contribution in the value of the non asymptotic error probability. This explains the big difference between outage and non asymptotic error probabilities for low rates. When $R$ increases, the outage probability takes into account the tail of the SNR distribution and the difference with the non asymptotic error probability reduces.

\section{CONClusion And Perspectives}

In this paper, we have investigated the impact of the nonasymptotic coding theory from a practical wireless communication point of view. In particular, we have studied the gap between the Shannon Pareto front on the EE-SE tradeoff and the real achievable tradeoff when finite-length codewords are employed in AWGN channels. The investigation reveals an increasing gap between the asymptotic fundamental limit 


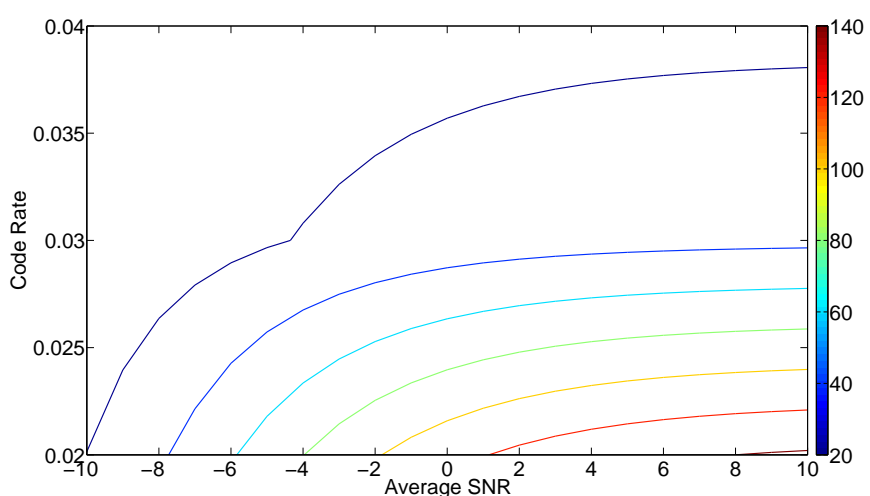

(a) $n=200, m=6$, low rates

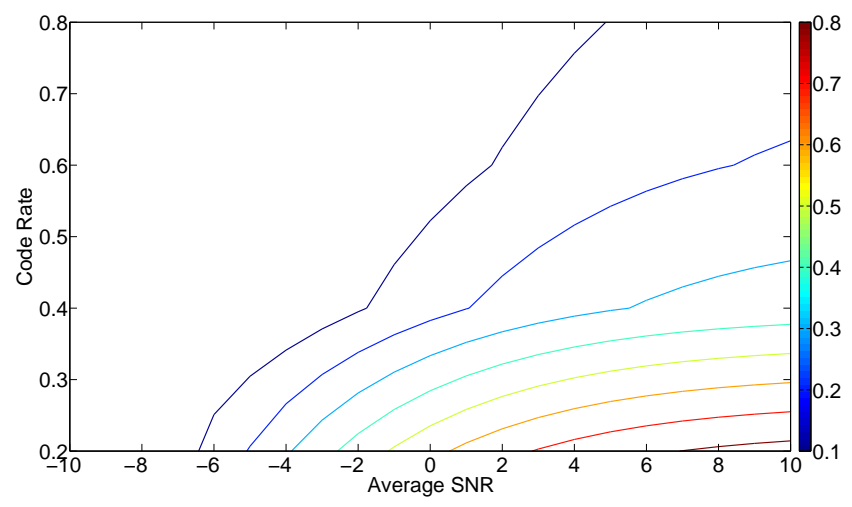

(b) $n=200, m=6$, moderate to high rates

Fig. 6. Relative difference between the outage and the average error probabilities in Nakagami $-m$ block fading vs the SNR and the rate.

(Shannon theory) and the non-asymptotic achievable EE-SE tradeoff when the error probability is decreasing. Moreover, the outage probability has also been investigated in Ricean and Nakagami- $m$ block fading channels, where the definition using the Shannon capacity formula can largely underestimate the outage probability compared to a non-asymptotic analysis, particularly for low rates. These results may be of particular interest for the study of the fundamental performance of future IoT/M2M networks, wherein low rates and short packets are envisaged to be the key characteristics.

\section{REFERENCES}

[1] C. Shannon, "A Mathematical Theory of Communication," Bell System Technical Journal, vol. 27, pp. 379-423, 623-656, July, October 1948.

[2] D. Tse and P. Viswanath, Fundamentals of Wireless Communication. Cambridge University Press, 2005.

[3] A. Feinstein, "A New Basic Theorem of Information Theory," Transactions of the IRE Professional Group on Information Theory, vol. 4, no. 4, pp. 2-22, September 1954.

[4] R. Gallager, "A Simple Derivation of the Coding Theorem and some Applications," IEEE Transactions on Information Theory, vol. 11, no. 1, pp. 3-18, Jan 1965.

[5] Y. Polyanskiy, H. Poor, and S. Verdu, "Channel Coding Rate in the Finite Blocklength Regime," IEEE Transactions on Information Theory, vol. 56, no. 5, pp. 2307-2359, May 2010.

[6] S. Verdu, "Non-Asymptotic Achievability Bounds in Multiuser Information Theory," in Proc. 50th Annual Allerton Conference on Coтmunication, Control, and Computing, Oct 2012, pp. 1-8.
[7] Y. W. Huang and P. Moulin, "Finite Blocklength Coding for Multiple Access Channels," in Proc. IEEE International Symposium on Information Theory, July 2012, pp. 831-835.

[8] V. Y. F. Tan and O. Kosut, "On the Dispersions of Three Network Information Theory Problems," in Proc. 46th Annual Conference on Information Sciences and Systems, March 2012, pp. 1-6.

[9] E. MolavianJazi, "A Unified Approach to Gaussian Channels with Finite Blocklength,” Ph.D. dissertation, University of Notre Dame, July 2014.

[10] W. Yang, G. Durisi, T. Koch, and Y. Polyanskiy, "Quasi-Static SIMO Fading Channels at Finite Blocklength," in Proc. IEEE International Symposium on Information Theory Proceedings, July 2013, pp. 15311535.

[11] _ "Block-Fading Channels at Finite Blocklength," in Proc. of the Tenth International Symposium on Wireless Communication Systems, Aug 2013, pp. 1-4.

[12] _- "Quasi-Static Multiple-Antenna Fading Channels at Finite Blocklength," IEEE Transactions on Information Theory, vol. 60, no. 7, pp. 4232-4265, July 2014.

[13] J. Hoydis, R. Couillet, and P. Piantanida, "The Second-Order Coding Rate of the MIMO Quasi-Static Rayleigh Fading Channel," IEEE Transactions on Information Theory, vol. 61, no. 12, pp. 6591-6622, Dec 2015.

[14] B. Makki, T. Svensson, and M. Zorzi, "Finite Block-Length Analysis of the Incremental Redundancy HARQ," IEEE Wireless Communications Letters, vol. 3, no. 5, pp. 529-532, Oct 2014.

[15] T. L. Marzetta and B. M. Hochwald, "Capacity of a Mobile MultipleAntenna Communication Link in Rayleigh Flat Fading," IEEE Transactions on Information Theory, vol. 45, no. 1, pp. 139-157, 1999.

[16] P. Mary, M. Dohler, J.-M. Gorce, G. Villemaud, and M. Arndt, "BPSK Bit Error Outage over Nakagami-m Fading Channels in Lognormal Shadowing Environments," IEEE Communications Letters, vol. 11, no. 7, pp. 565-567, July 2007.

[17] _ _M-ary Symbol Error Outage over Nakagami-m Fading Channels in Shadowing Environments," IEEE Transactions on Communications, vol. 57, no. 10, pp. 2876-2879, 2009.

[18] P. Mary, M. Dohler, J.-M. Gorce, and G. Villemaud, "Packet Error Outage for Coded Systems Experiencing Fast Fading and Shadowing," IEEE Transactions on Wireless Communications, vol. 12, no. 2, pp. 574-585, February 2013. 